\title{
Decyphering "the MASTER CODE ®” Structure and Discovery of a Periodic Invariant Unifying 160 HIV1/HIV2/ SIV Isolates Genomes
}

\author{
Jean-claude Perez* \\ Retired Interdisciplinary Researcher (IBM), Francee
}

Received: July 17, 2017; Published: July 19, 2017

*Corresponding author: DR Jean-claude Perez, Maths and Computer Science Retired interdisciplinary researcher (IBM), 7 avenue de terre-rouge F33127 Martignas, Bordeaux metropole, France, Tel: 33 0781181112; Email: jeanclaudeperez2@gmail.com/jeanclaudeperez3@free.fr

Keywords: Genomes Invariant; Periods; Genomes Unification Dr

\section{Methods}

\section{Master Code}

Starting from the fundamental basic level of Bio-Atoms atomic weights, we discovered a numeric code common to the 3 Genetics worlds: DNA, RNA and amino acids. Coding Genomics (DNA double strands) and "Virtual Proteomics"(corresponding amino acids translations of DNA), we obtain specific couples of curves called Master Code signatures; in all cases both curves are highly correlated, meanwhile, there are hierarchies between these Genomics/Proteomics coupling ratios: Analyzing and comparing Master Codes in Achaea's, Bacteria's, Viruses, and Eukaryotes reveals function (actives sites, SNPs locations etc), genomes integrity, differentiation and evolution new information [1-5]. We have data suggesting the Electrical nature of this Law. Specifically in the case of Human Genome, we prove triple quasi UNIFICATION between:

A. The 2 Genomics and virtual Proteomics bio-chemical materializations.

B. The 3 possible codons reading frames.

C. The 4 combinatorial possibilities readings directions of both DNA strands.

In fact, we demonstrate the evidence of a super UNIFIED information level including both Genomics and Proteomics worlds. We thus analyzed the 285 and the 66 millions base-pairs of human chromosomes 1 and 20 (more than $10 \%$ of the whole draft human genome). By analysing both chromosomes in independent units of 1 Mbps, there are $(285+66) \times 2 \times 3 \times 4$ independent sequences which were studied (nearly 8.4 billion base-pairs). Then, there appears a master code structuring the complete draft human chromosomes 1 and 20 with an average Genomics/Proteomics coupling ratio of $97 \%$. Then another level of reading reveals embedded long distance periodic structures throughout whole genomes [6-12]. These PERIODS, expressed in Codons numbers multiples, characterize globally specific Genomes: there are independent with classical nucleotides repeats. A possible meaning of this discovery could be related with DNA meta-structure at physical and spatial levels (DNA molecule on-rolling up). These discoveries constitutes a "shadedlanguage" called the "universal genomic code". This fundamental discovery was first published in the book (4) then summarized by the main article (9), and was generalized to all genomes available today: archaeas, bacteria's,viruses, worm, fly, Arabidopsis and the complete draft humangenome.

\section{Results}

\section{What about AIDS retroviruses genomes?}

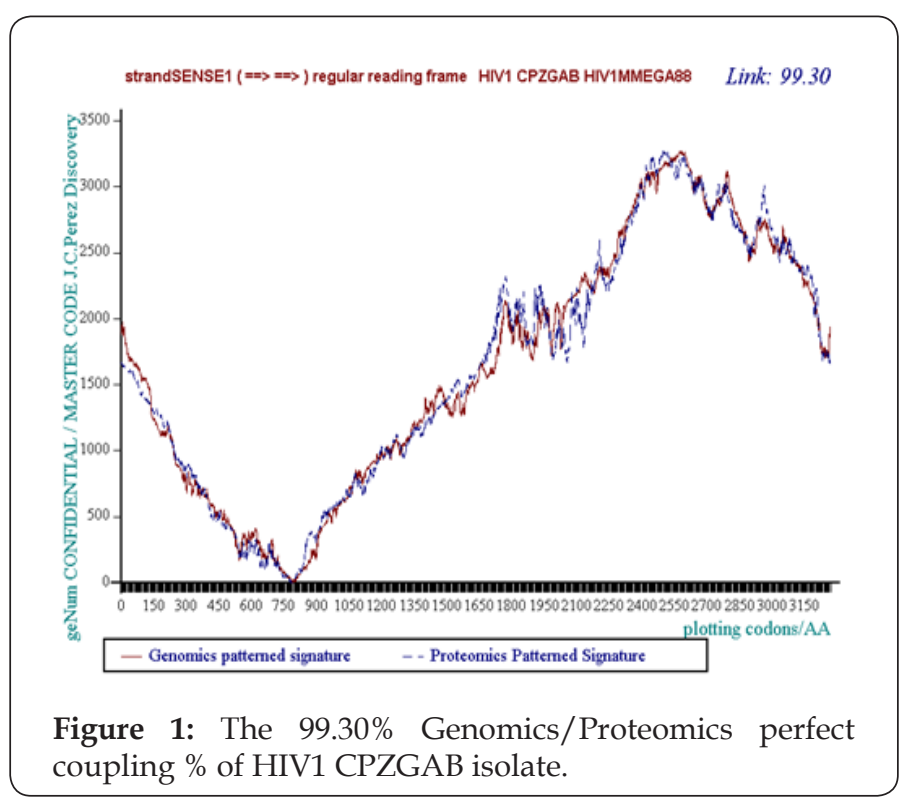


Like other viruses genomes, the HIV/SIV genomes provide Master Code coupling and periods. Meanwhile, the coupling is worse than in the case of the human genome. We have analyzed the master code of the 160 HIV1/HIV2/SIV genomes available from the LOS ALAMOS AIDS database. Our innovative results provide for each specific AIDS genome isolate:

A. MASTER CODE Genomics/Proteomics coupling signature (see (Figure1)- HIV1 CPZGAB isolate).

B. EVIDENCE of META-PERIODS overlapping whole genomes (see (Figure 2)- HIV1 NDK isolate).

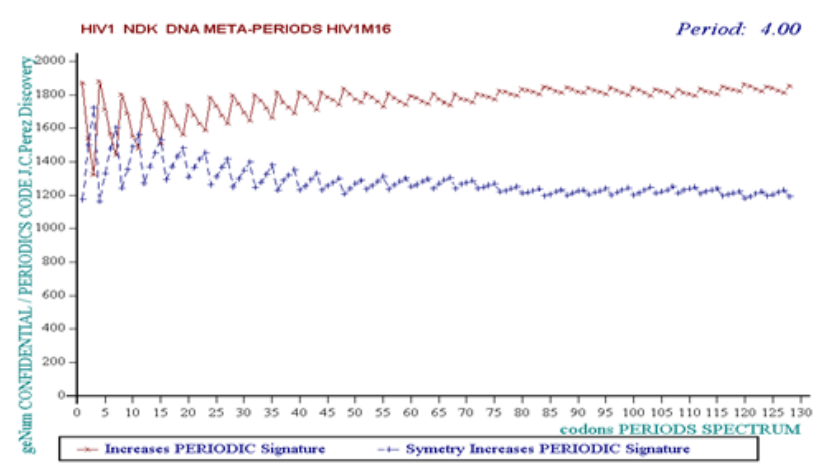

Figure 2: The perfect 4-Codons META-PERIOD of HIV1 NDK isolate.

C. MASTER CODE Genomics/Proteomics average coupling for the better codons reading frame of the 160 complete genomes: $92.2 \%$.

\section{Details}

Average results for the 89 HIV1 isolates: 92.05\% / Average results for the 28 HIV2 isolates: 91.31\% / Average results for the 43 SIV isolates: 93.09\% [13-15]. EVIDENCE of a common 4-codons META-PERIOD (12 nucleotides) Invariant Unifying all the $160 \mathrm{HIV} / \mathrm{SIV}$ referenced Genomes Isolates. Evidence of small PERIOD-4 variations in some specific isolates linked with possible heterogeneity/differentiation ways related to these Genomes [16].

\section{Conclusion}

The discovery of an invariant of very high level which either common to the totality of the 160 known HIV1, HIV2 and SIV variants Genomes constitutes a new fact. The study of the fluctuations of certain genomes around this PERIOD-4 reference could make it possible to build universal vaccines or therapies. The fact that this PERIOD-4 is specific to all the HIV retroviruses, differentiating them with other viruses, bacteria's or human genomes, could be used towards an universal targeted AIDS vaccine.

\section{References}

1. Perez JC (1991) Chaos DNA and neuro-computers: a golden link. Speculations in Science and Technology 14: 336-346.

2. Marcer PJ (1992) Order and chaos in DNA - the Denis Guichard Prizewinner: Jean-Claude Perez. Kybernetes 21: 60-61.

3. Perez JC (1997) L'adn Décrypté. Resurgence publisher Liege, Belgium.

4. Perez JC (2009) Codex Biogenesis. Resurgence, Liege, Belgium.

5. Perez JC (2010) Codon Populations in Single-Stranded Whole Human Genome DNA Are Fractal and Fine-Tuned by the Golden Ratio 1.618. Interdiscip Sci 2(3): 228-240.

6. Perez JC (2011) Caminos Interdisciplinaios. Seminario CLAVE_INTER, Espacio Interdisciplinario, Universidad de la Republica Montevideo, Uruguay.

7. Perez JC (2011) Decoding Non-Coding DNA Codes: Human Genome Meta-Chromosomes Architecture. BIT Life Sciences, 3rd Annual World Vaccine Congress, Beijing, China.

8. Perez JC (2013) The 3 Genomic Numbers Discovery: How Our Genome Single-Stranded DNA Sequence Is Self- Designed as a Numerical Whole. Applied Mathematics 4: 37-53.

9. Perez J (2015) Deciphering Hidden DNA Meta-Codes -The Great Unification \& Master Code of Biology. J Glycomics Lipidomics 5:131.

10. Perez JC (2017) Fractal Self-similarity, Scale Invariance and Stationary waves Codes Architecture Human Chromosomes DNA sequences.

11. Perez JC (2017) Sapiens Mitochondrial DNA Genome Circular Long Range Numerical Meta Structures are Highly Correlated with Cancers and Genetic Diseases mtDNA Mutations. J Cancer Sci Ther 9:512-527. doi: 10.4172/1948-5956.1000469 https://www.omicsonline.org/ open-access/sapiens-mitochondrial-dna-genome-circular-long-rangenumericalmeta-structures-are-highly-correlated-with-cancers-andgenetic-disea-1948-5956-1000469.php?aid=90737.

12. perez, DUF1220 Homo Sapiens and Neanderthal fractal periods architectures breakthrough(2017)SDRP Journal of Cellular and Molecular Physiology 1(1)http://www.siftdesk.org/article-details/ DUF1220\%20Homo\%20Sapiens $\% 20$ and $\% 20$ Neanderthal $\% 20 \% 20$ fractal $\% 20$ periods $\% 20$ architectures $\% 20$ breakthrough $/ 184$ ?

13. Perez JC (2017) Humans and Primates Chromosomes4 Fractal CODES: periodic stationnary waveforms charaterizing and differenciating Neanderthal and Sapiens whole chromosomes DNA sequences.

14. Perez JC (2017) Global and long range fractal differences between sapiens and neanderthal genomes.

15. Montagnier L (2017) Water Bridging Dynamics of Polymerase Chain Reaction in the Gauge Theory Paradigm of Quantum Fields. Water 9(5): 339.

16. Perez JC (2017) The Human Genome Optimum (HGO): towards a Universal Law controlling all human Cancer Chromosome LOH Deletions (Loss Of Heterozygosity). 


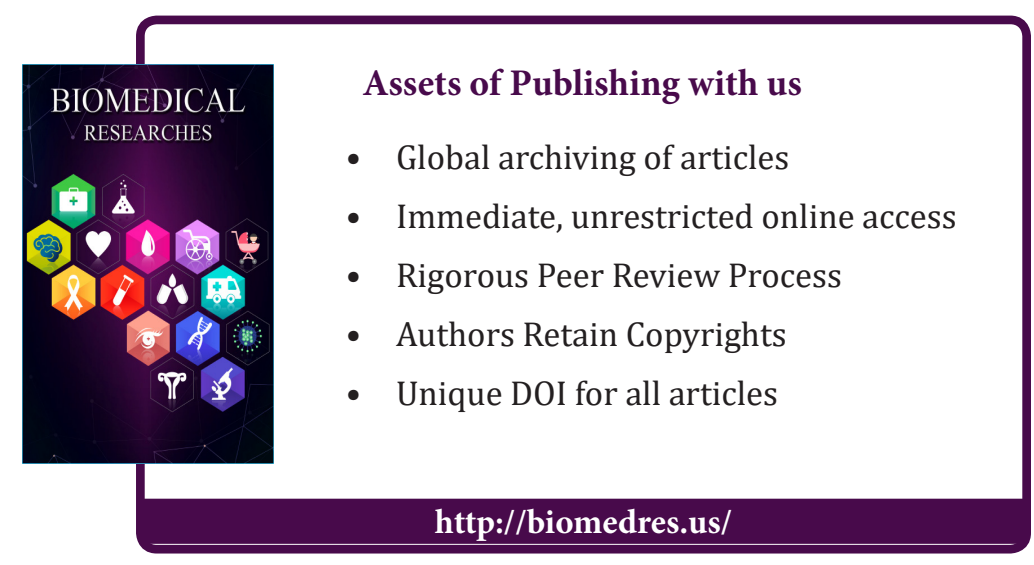

